\title{
A IMPORTÂNCIA DA ESTATÍSTICA DESCRITIVA NO SETOR DE QUALIDADE EM UMA EMPRESA DE GARRAFAS PLÁSTICAS
}

Ciro Ávila Machado Ferreira - ciro.ferreira@alunos.unis.edu.br Filipe de Oliveira Proença - filipe.proenca@alunos.unis.edu.br Guilherme Reis Portilho - guilherme.portilho@alunos.unis.edu.br Victor Hugo Silva Lucas - victor.lucas@alunos.unis.edu.br Mário César Fialho de Oliveira - mario.oliveira@professor.unis.edu.br

\section{RESUMO}

Os métodos estatísticos foram desenvolvidos como uma mistura de ciência, tecnologia e lógica para a solução e investigação de problemas em várias áreas do conhecimento.No setor da indústria, os engenheiros utilizam técnicas estatísticas com o objetivo de acompanhar o controle da qualidade dos produtos dentro de um determinado nível de aceitação.Neste artigo foi realizada uma análise quantitativa em uma pesquisa de campoem uma indústria de garrafas plásticas localizada em Miraí/MG a fim de analisar os impactos das medidas-resumo mais usuais da estatística descritivas para o setor industrial, principalmente, a variância e o desvio padrão. $\mathrm{O}$ aspecto estudado foi a gramatura de uma amostra de garrafas de polietileno e os trabalhos de análises estatísticas foram realizados através doSoftware Maple 17. Ao final do estudo, notou-se importância do estudo da variabilidade. Dentro das medidas-resumo calculadas, pode-se destacar o impacto negativo que uma alta taxa de variância acarreta a linha produtiva de uma empresa desse segmento. Um valor alto para o desvio-padrão implica em uma grande diversidade de valores para as gramaturas, provocando uma ruptura do padrão de qualidade. Portanto, para o setor industrial é primordial a pesquisa e a aplicação da estatística descritiva com o objetivo de fornecer informações para um diagnóstico mais eficaz na prevenção e detecção de falhas/defeitos, identificando suas causasem tempo real, o que, consequentemente, auxilia no aumento da produtividade/resultados da organização, evitando desperdícios de matéria-prima, insumos, produtos, entre outros.

Palavras-chave: Qualidade; Estatística; Embalagens Plásticas; Garrafas.

\begin{abstract}
Statisticalmethodsweredeveloped as a mixtureofscience, technologyandlogic for thesolutionandinvestigationofproblems in variousareasofknowledge. In theindustry sector, engineers use statisticaltechniques in orderto monitor thequalitycontrolofproductswithin a certainlevelofacceptance. In thisarticle, quantitativeresearchandfieldresearchwerecarried out in

in

Miraí/MG

in
\end{abstract}


ordertoanalyzetheimpactsofdescriptivestatisticsmost usual summarymeasures for the industrial sector, mainly, varianceand standard deviation. The studiedaspectwasthegrammageof ofpolyethylenebottlesandthestatisticalanalysisworkwascarried out usingthe Maple 17 software. At theendofthestudy, theimportanceofthestudyofvariabilitywasnoted. Withinthecalculatedvariables, the negative impactthat a high variance rate hasontheproductionlineof a company in thissegmentcanbehighlighted. A high value for thisvariableimplies a greatdiversityofvalues for grammages, causing a rupture in thequality standard. Therefore, for the industrial sector it isessentialtoresearchandapplydescriptivestatistics in ordertoprovideinformation for a more effectivediagnosis in thepreventionanddetectionoffailures/defects, identifyingtheir causes in real time, whichconsequently helps in theincreasingtheorganization'sproductivity/results, avoidingwasteofrawmaterials, inputs, products, amongothers.

Keys:Quality; Statistic; Plasticpackages; Bottles.

\section{INTRODUÇÃO}

Durante o século XX, segundo o autorSalsburg (2009), a estatística revolucionou a ciência através do fornecimento de modelos úteis que sofisticaram o processo de pesquisa na direção de melhores parâmetros de investigação, permitindo auxiliar a tomada de decisões nas políticas socioeconômicas. Para Stigler (1986), os métodos estatísticos foram desenvolvidos como uma mistura de ciência, tecnologia e lógica para a solução e investigação de problemas em várias áreas do conhecimento.

A estatística pode ser considerada como uma ciência, um método ou uma arte. É vista como ciência quando, baseando-se em suas teorias, analisa-se grandes conjuntos de dados, independentemente da natureza destes, sendo autônoma e universal. É conhecida como um método quando serve de instrumento particular a uma determinada ciência(como na agronomia,na biologia, na física, na medicina ou na psicologia). Finalmente, é encarada como arte quando é aplicada visando à construção de modelos para representar a realidade (LOPES, 2005).

Assim, entre todas essas classificações, a estatística é responsável pelo planejamento de experimentos, interpretação dos dados obtidos através de pesquisas de campo e apresentação de resultados de maneira a facilitar a tomada de decisões por parte do pesquisador ou do gestor. 
No setor da indústria, os engenheiros utilizam técnicas estatísticas com o objetivo de acompanhar o controle da qualidade dos produtos dentro de um determinado nível de aceitação. A importância dessa utilização é destacada por Rao (19992, apud ESTATÍSTICAS..., 2010):

É experiência comum no mundo inteiro que, nas indústrias onde os métodos estatísticos são explorados, a produção aumentou em cerca de dez a cem por cento, sem nenhum investimento adicional nem expansão industrial. Neste sentido, o conhecimento estatístico é considerado um recurso nacional. Não é surpreendente que um livro recente sobre invenções modernas liste o controle estatístico de qualidade como uma das grandes invenções tecnológicas do século XX. De fato, raramente houve uma invenção tecnológica como o controle estatístico de qualidade, que é tão amplo em aplicações, mas tão simples em teoria, que é tão efetivo em resultados, mas tão fácil de adotar e que gera um retorno tão alto, mas requer um investimento tão pequeno.

No setor industrial é primordial a pesquisa e a aplicação da estatística descritiva, com o objetivo de fornecer informações para um diagnóstico mais eficaz na prevenção e detecção de falhas/defeitos, identificando suas causasem tempo real, o que, consequentemente, auxilia no aumento da produtividade/resultados da organização, evitando desperdícios de matériaprima, insumos, produtos, entre outros.

\section{METODOLOGIA}

Para o desenvolvimento deste artigo, realizou-se uma pesquisa bibliográfica através da análise de fontes de pesquisa secundária (livros, manuais, artigos e monografias) e uma pesquisa quantitativa através da análise estatísticaunivariada oriunda da pesquisa de campo em uma empresa de embalagens plásticas localizada na cidade de Miraí em Minas Gerais.

O estudo de caso é uma maneira de investigação de um contexto empírico através de procedimentos específicos. Para Yin (2001), um estudo de caso é uma investigação empírica que investiga um fenômeno contemporâneo dentro de um contexto de vida real, especialmente quando os limites entre o fenômeno e o contexto não estão claramente definidos. Dessa forma, essa abordagem permite realizar pesquisas in loco, facilitando estudos organizacionais e gerenciais que contribuem para a essência do tema, aumentando o conhecimento científico sobre o assunto e ainda proporciona melhorias, resultados operacionais e estratégicos das empresas em estudo.

Para a realização dos trabalhos de análise dos dados foi utilizado o Software Maple 17, sendo os resultados apresentados de forma gráfica e tabulada. Na sequência, serão apresentados os resultados encontrados.

\section{A EMPRESA}


O estudo abordado no presente artigo foi realizado em uma indústria de embalagens plásticas que se destaca no processo de sopro de garrafas PET e de Polietileno produzidas para atender, principalmente, os segmentos: lácteo, água mineral, suco, dentre outros. Além das garrafas padrões de linha, possui um setor de desenvolvimento de modelos (moldes) exclusivos de acordo com a necessidade do cliente ou do mercado.

Em relação a sua estrutura, possui um amplo parque industrial, equipamentos de sopro de alta tecnologia divididos entre as linhas de PET e Polietileno, frota própria de caminhões, um espaço reservado para matéria-prima e outro para produto acabado, e escritórios para cada setor (administrativo, comercial, financeiro, fiscal, contábil, compras, produção, estoque, qualidade e departamento pessoal).

Para o estudo de caso, foi realizada a análise da gramatura de uma amostra de 55 garrafas de polietileno a fim de estudar a média populacional, a moda, a mediana, a variância, o desvio padrão, a curtose e o intervalo de confiança dessa amostra através do Software Maple 17.

\section{REVISÃO DE LITERATURA}

\section{Definição de qualidade}

Dentro da literatura existe uma diversidade de definições diferentes para o termo qualidade. Comparando os diversos pontos de vistas, a qualidade é o atendimento às necessidades atuais ou futuras do cliente (Deming apud Nora, 2003), a satisfação das aspirações do usuário e adequação ao uso (Juranapud Nora, 2003), a conformidade com os requisitos (Crosby apud Nora, 2003) e a busca contínua das necessidades do cliente visando sua satisfação (Ishikawa apud Nora, 2003).

Analisando-se a multiplicidade de definições para o termo qualidade, percebe-se que existe um ponto em comum: a visão do cliente. Dessa maneira, pode ser considerado um conceito relativo, pois, depende do ponto de vista do consumidor. Buscando abordar todas as ramificações da qualidade, pode-se defini-la utilizando cinco abordagens diferentes: abordagem transcendental, abordagem baseada no produto, abordagem baseada no usuário, abordagem baseada na produção e abordagem baseada no valor (Garvin, 2002). 


\begin{tabular}{|c|l|}
\hline $\begin{array}{c}\text { Abordagem } \\
\text { transcendental: }\end{array}$ & $\begin{array}{l}\text { qualidade é entendida como sinônimo de excelência } \\
\text { inata, e assim não pode ser medida com precisão, } \\
\text { sendo apenas reconhecida pela experiência; }\end{array}$ \\
\hline $\begin{array}{c}\text { Abordagem baseada } \\
\text { no produto: }\end{array}$ & $\begin{array}{l}\text { qualidade relaciona-se ao maior número e melhores } \\
\text { características que um produto apresenta. Nessa } \\
\text { abordagem, a qualidade é vista como uma variável } \\
\text { precisa e mensurável, com foco na durabilidade, de } \\
\text { tal forma que alta qualidade implica em alto custo; }\end{array}$ \\
\hline $\begin{array}{c}\text { Abordagem baseada } \\
\text { no usuário: }\end{array}$ & $\begin{array}{l}\text { qualidade é o atendimento às necessidades e } \\
\text { preferências do consumidor. Nessa abordagem, } \\
\text { presume-se que a qualidade está diante dos olhos } \\
\text { dos consumidores, portanto é altamente subjetiva; }\end{array}$ \\
\hline $\begin{array}{c}\text { Abordagem baseada } \\
\text { na produção: }\end{array}$ & $\begin{array}{l}\text { o conceito de qualidade nessa abordagem é } \\
\text { sinônimo de conformidade com especificações e e } \\
\text { adequação da fabricação às exigências do projeto. } \\
\text { Ainda, a melhoria da qualidade equivale à redução } \\
\text { no número de desvios, levam a menores custos; }\end{array}$ \\
\hline $\begin{array}{c}\text { Abordagem baseada } \\
\text { no valor: }\end{array}$ & $\begin{array}{l}\text { qualidade é o desempenho ou conformidade a um } \\
\text { preço ou custo aceitável. }\end{array}$ \\
\hline & Tabela 1. Fonte: Gavin, 2002. \\
\hline
\end{tabular}

Para Garvin (2002), a qualidade ainda possui diferentes faces: performance (mede o desempenho), funcionalidade do produto (conjunto de funções que complementam a oferta do produto), fiabilidade (probabilidade de o produto deixar de funcionar em um determinado período de tempo), conformidade (adequação às especificações), durabilidade (tempo de vida do produto), serviço (agilidade e eficiência no reparo do produto), aparência (estética) e imagem (percepção subjetiva).

Assim como a Teoria da Totalidade de Bohm (2008) - que afirma que quanto maior a disponibilidade de elementos no processo de tomada de decisão, maiores as chances de alcançar resultados positivos - a coexistência desses diversos conceitos, para o termo qualidade, estimula a melhoria da comunicação entre fornecedores, clientes e áreas distintas de uma organização. Portanto, conclui-se que a qualidade se tornou primordial para atingir patamares diferenciados dentro do mercado competitivo, reforçando a importância do estudo da gestão da qualidade.

Considerando a evolução do mercado e o aumento da competitividade devido ao maior número de concorrentes, além da necessidade de desenvolvimento de novos produtos em uma velocidade cada vez maior, a busca pela qualidade de produtos e serviços se tornou prioridade para as organizações (Yang, 2008). 


\title{
Definição de estatística e sua aplicabilidade
}

A estatística pode ser definida como um conjunto de métodos e técnicas que envolve todas as etapas de uma pesquisa, desde o planejamento, coordenação, levantamento de dados por meio de amostragem ou censo, aplicação de questionários, entrevistas e medições com a máxima quantidade de informação possível para um dado custo, até a consistência, processamento, organização, análise e interpretação de dados para explicar fenômenos socioeconômicos; inferência, cálculo do nível de confiança e do erro existente na resposta para uma determinada variável e disseminação das informações.

Para Matsushita (2010),

\begin{abstract}
o que se entende, modernamente, por Estatística ou Ciência Estatística é muito mais do que um conjunto de técnicas úteis para algumas áreas isoladas ou restritas da ciência. Por exemplo, ao contrário do que alguns imaginam, a estatística não é um ramo da matemática onde se investigam os processos de obtenção, organização e análise de dados sobre uma determinada população. Também não se limita a um conjunto de elementos numéricos relativos a um fato social, nem a tabelas e gráficos usados para o resumo, a organização e apresentação dos dados de uma pesquisa, embora este seja um aspecto da estatística que pode ser facilmente percebido no cotidiano.
\end{abstract}

Baseando-se em Rao (1997), um dos mais importantes estatísticos do século XX, a estatística pode ser definida, de forma simples e objetiva, pela equação: conhecimento incerto + conhecimento sobre a incerteza $=$ conhecimento útil. Portanto, a estatística visa analisar os dados disponíveis e que estão sujeitos a certo grau de incerteza no planejamento e obtenção de resultados.

Inesul (2007) pontua que foi somente no século XIX que a estatística começou a ganhar importância nas diversas áreas do conhecimento. A partir do século seguinte, começou a ser implantada nas grandes organizações, quando os japoneses começaram a falar em qualidade total, surgindo a estatística moderna, considerada uma disciplina. A partir desse marco, evoluiu de forma significativa, passando a ser utilizada em diferentes setores da sociedade a fim de obter informações a partir do levantamento de dados com base em métodos de amostragem complexos. Os avanços no campo da TI (Tecnologia da Informação), envolvendo todas as atividades e soluções providas por recursos de computação (hardwares e softwares), a partir da metade do século XX e atualmente, tendo como consequências o aumento significativo da capacidade de produzir, armazenar e transmitir informações, associados ao crescimento acentuado da demanda por informações num mundo globalizado, vêm exigindo da estatística avanços paralelos no desenvolvimento de metodologias e novos indicadores cada vez mais complexos, que exigem equipamentos modernos, softwares estatísticos e técnicos capacitados. 
A utilidade da estatística se expressa no seu uso, considerando-se que grande parte das hipóteses científicas, independentemente da área, precisa passar por um estudo estatístico para ser aceita ou rejeitada. Na área médica, por exemplo, nenhum medicamento, vacina ou procedimento pode ser disponibilizado para o mercado se não tiver sua eficácia estatisticamente comprovada. O exemplo mais atual é a produção e a disponibilização em massa de uma vacina eficaz para o SARS-Cov-2 (COVID 19), onde testes científicos e estatísticos são realizados a todo momento a fim de avaliar a eficácia da imunização. $\mathrm{O}$ grande volume de informações produzidas pelo mundo moderno (pesquisas por amostragem, censos, internet, mercado financeiro) precisa ser analisado criteriosamente. Essas análises utilizam as diversas técnicas estatísticas. A rigor, onde houver incerteza esta ciência pode ser empregada. Desse modo, todas as áreas do conhecimento humano a requerem como instrumento de análise de dados (PIMENTEL, 2009).

\section{Pensamento estatístico e melhoria da qualidade}

A percepção dos princípios do pensamento estatístico e a melhor de utilizá-los buscando a melhoria da qualidade, pode ser um diferencial que contribui para a efetividade máxima do gerenciamento (GRIGG; WALLS, 2007b; MAKRYMICHALOS et al., 2005). O principal obstáculo é descobrir quais são os benefícios da abordagem estatística que contribuem de forma significativa para o processo de tomada de decisão.

Conforme expõem Britz et al. (2000), o pensamento estatístico possibilita que as decisões de modificações no processo sejam baseadas em elementos concretos e não em informações subjetivas como as percepções ou experiências passadas. Coleman e Stewardson (2002) corroboram a importância dos dados como suporte para a melhoria da qualidade, visto que eles fornecem evidências que propiciam um controle mais efetivo e a diminuição da variabilidade. Todavia, para que os dados apresentem informações confiáveis, tanto sua coleta, quanto sua análise e apresentação, devem seguir um planejamento e uma execução fundamentados estatisticamente (GRIGG; WALLS, 2007b).

Para Santos e Martins (2008), o pensamento estatístico representa um pano de fundo para a melhoria efetiva dos processos, por ser uma filosofia que permite uma visão mais holística. Com a análise mais criteriosa e profunda dos processos, se produz um entendimento melhor sobre as fontes de variação que precisam ser modificadas ou remodeladas para, em seguida, focar na variação. Através desse eixo, identifica-se a necessidade mais proeminente da utilização dos métodos estatísticos descritivos a fim de promover a melhoria contínua e assegurar os ganhos almejados. 


\section{DISCUSSÃO E RESULTADOS}

Para a análise estatística desse artigo, os autores utilizarem-se do processo de amostragem aleatória simples através do qual foram segregadas 55 garrafas de polietileno (figura 1) todas com a mesma volumetria. Posteriormente, foi verificada a gramatura de cada unidade através da aferição da massa em uma balança de precisão (figura 2) com o objetivo de explorar a média populacional, a moda, a mediana, a variância, o desvio padrão, a curtose e o intervalo de confiança dessa amostra através do Software Maple 17.

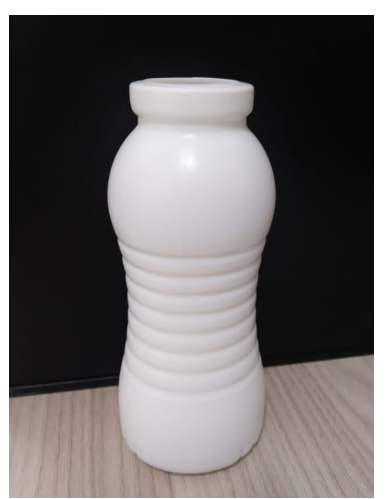

Figura 1. Modelo da garrafa analisada

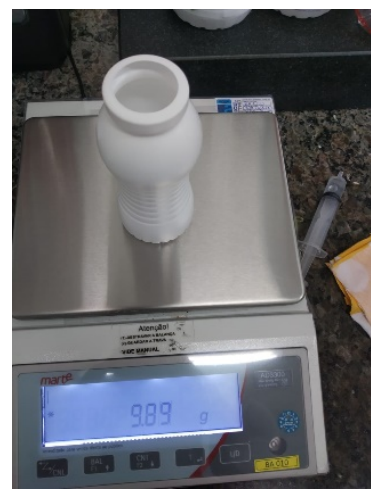

Figura 2. Processo de aferição da massa.

Através do estudo desses dados e da utilização do Maple 17, foi possível definir as medidas-resumo e concluir os dados que seguem abaixo.

\begin{tabular}{|c|c|}
\hline Medidas-resumo & Resultado \\
\hline Média Populacional & 9,792727273 \\
\hline Moda & 9,9 \\
\hline Mediana & 9,9 \\
\hline Variância & 0,059575758 \\
\hline Desvio Padrão & 0,244081457 \\
\hline Curtose & 2,999999999 \\
\hline Média da Amostra & 9,83 \\
\hline $\begin{array}{c}\text { Desvio Padrão da } \\
\text { Amostra }\end{array}$ & 0,222463021 \\
\hline
\end{tabular}


Tabela 2. Criada pelos autores. Fonte: Maple 17.

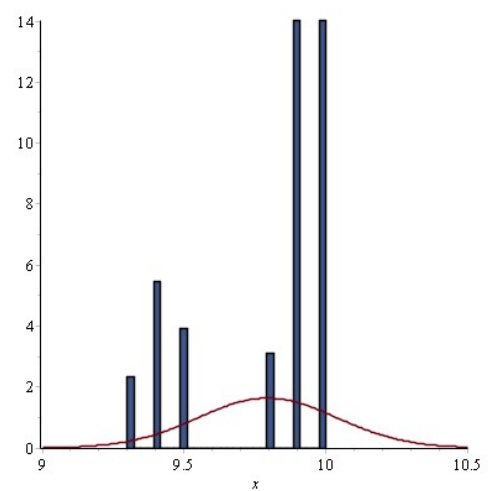

Figura 3. Gráfico Curtose.

\section{Média da amostra e desvio padrão da amostra}

\begin{tabular}{|c|c|}
\hline Intervalo gramatura (g) & Quantidade de garrafas \\
\hline $9<\mathrm{x}<9.5$ & 10 \\
\hline $9.2<\mathrm{x}<10.1$ & 55 \\
\hline $9.5<\mathrm{x}<10$ & 22 \\
\hline $9<\mathrm{x}<10$ & 37 \\
\hline $9.3<\mathrm{x}<9.8$ & 12 \\
\hline
\end{tabular}

Tabela 3. Intervalo gramatura $(\mathrm{g}) \times$ quantidade de garrafas.

Média $(X)=9,830$

Desvio Padrão (s) $=0,222463021462821$

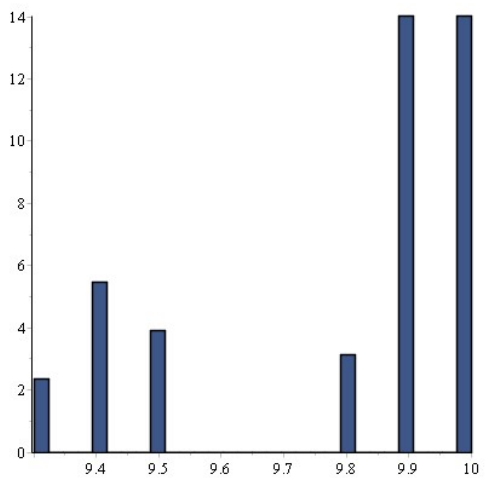

Figura 4. Gráfico quantidade de garrafas x gramatura

Intervalo de confiança

A determinação do intervalo de confiança da média permite conhecer o intervalo que contém a média populacional a um determinado nível de confiança (Fonseca e Martins, 1982, p. 162).

O intervalo de confiança da média pode ser calculado a partir da estatística t, conforme segue (Fonseca e Martins, 1962, p. 165): 


$$
t_{n-1}=\frac{\bar{X}-\mu}{S / \sqrt{n}}
$$

Onde $\mu$ é a média populacional e n é o tamanho da amostra. No caso deste artigo e conforme mostrado anteriormente:

$$
\begin{gathered}
\mu=9,79272727272727 \\
n=55
\end{gathered}
$$

Sendo assim, foi elaborado o gráfico do intervalo de confiança.

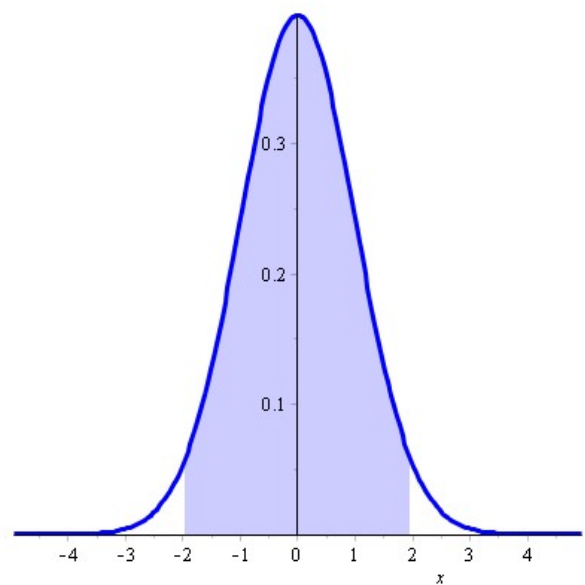

Figura 5. Gráfico Intervalo de Confiança.

\section{Média de uma amostragem maior}

Tendo em vista, a importância de testar e ratificar as análises estatísticas, os autores deste artigo simularam no Software Maple uma amostra de 10.000 unidades de garrafas dentro das mesmas condições da amostra anterior (conforme figura 7). Dessa forma, foi possível ampliar e analisar a situação de uma perspectiva mais abrangente.

$\begin{aligned} & = \\ & =\end{aligned}:=$ Random Variable $(\operatorname{Norm} \operatorname{mal}(9.5,0.5)):$

$>V:=\operatorname{Sample}(X, 10000)$

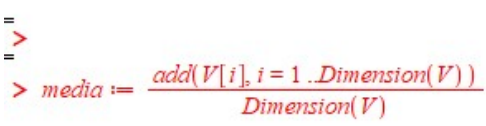

$>$ media $:=\frac{\operatorname{add}(V[i], i=1 . \operatorname{Dimension}(V))}{\operatorname{Dimension}(V)}$

$$
V:=\left[\begin{array}{c}
1 . .10000 \text { Vector }_{\text {row }} \\
\text { Data Type: float } \\
\text { Storage: rectangular } \\
\text { Order: Fortran_order }
\end{array}\right]
$$

media $:=9.50545470809803$

Figura 6. Amostra de 10.000 unidades. 


\section{CONCLUSÃO}

Mediante toda a revisão literária realizada e a observação in loco na empresa escolhida, notou-se o quanto é importante um trabalho sistêmico e que considere as análises estatísticas descritivas com o objetivo de se manter competitiva no mercado através da redução de custos, eliminação dos desperdícios, aumento da eficiência produtiva, além da fidelização do cliente através da manutenção de materiais de qualidade e da flexibilidade em se adequar as demandas que surgem a cada nova época.

Através do software Maple 17, foi possível identificar e analisar a importância do estudo da variabilidade da gramatura das garrafas. Dentro das medidas-resumo calculadas, pode-se destacar o impacto negativo que uma alta taxa de variância acarreta a linha produtiva de uma empresa desse segmento. Um valor alto para essa variável implica em uma grande diversidade de valores para as gramaturas, provocando uma ruptura do padrão de qualidade. Posteriormente, seria interessante uma nova investigação sob a ótica do consumidor, ponderando e analisando os impactos dessas medidas-resumo para o setor comercial/vendas, visto que essa variabilidade também pode incidir na qualidade e apresentação do produto dos clientes.

Portanto, para o setor industrial é primordial a pesquisa e a aplicação da estatística descritiva, principalmente em setores como os de qualidade e PCP (Programação e Controle da Produção), com o objetivo de fornecer informações para um diagnóstico mais eficaz na prevenção e detecção de falhas/defeitos, identificando suas causasem tempo real, o que, consequentemente, auxilia no aumento da produtividade/resultados da organização, evitando desperdícios de matéria-prima, insumos, produtos, entre outros.

Dessa forma, conclui-se que para atingir patamares cada vez mais elevados no mercado, superando novas metas de padrões de qualidade atendendo as necessidades dos consumidores e garantindo uma flexibilidade de adaptação às exigências internas e externas, as organizações devem possuir um setor de qualidade atento, empenhado e atualizado com as novas ferramentas de análise, planejamento e controle tanto gerenciais quanto operacionais e estatísticos.

\section{REFERÊNCIAS BIBLIOGRÁFICAS}

BOHM, D. Totalidade e a ordem implicada. São Paulo: Madras, 2008.

BRITZ, G. C. et al. Improving performance throughstatisticalthinking Milwaukee: ASQ Quality Press, 2000. 171 p. 
COLEMAN, S. Y.; STEWARDSON, D. J. Use ofmeasurementandchartstoinform management decisions. ManagerialAuditingJournal, v. 17, n. 1-2, p. 16-

19, 2002.Disponível em: <http://dx.doi.org/10.1108/02686900210412199>. Acesso em: 31 de maio de 2021.

COSTA JR, A.G. Avaliação do impacto da certificação ISO 9001:2000 no desempenhoorganizacional nas empresas do sul de Minas Gerais. Dissertação (Mestrado emEngenharia de Produção) - Universidade Federal de Itajubá, Itajubá, 2005.

ESTATÍSTICAS, atuação profisssional, formação, mercado de trabalho. Disponível em: $<$ http://www.portalsaofrancisco.com.br/alfa/estatisticas/estatisticas-2.php $>$. Acesso em: 14 de novembro de 2020.

Fonseca, J.S.; Martins, G.A. 1982. Curso de estatística. São Paulo, Editora Atlas. 286p.

GARVIN, D.A. Gerenciando a qualidade: a visão estratégica e competitiva. Rio deJaneiro: Qualitymark Editora, 2002.

GRIGG, N.; WALLS, L. The role ofcontrolcharts in promotingorganizationallearning: new perspectives from a food industrystudy. The TQM Magazine, v. 19, n. 1, p. 37-49, 2007b. Disponível em: <http://dx.doi.org/10.1108/09544780710720826>. Acesso em: 31 de maio de 2021.

INESUL. Inesul destaca a importância da estatística no mundo contemporâneo. 2007. Disponível em: $<$ http://www.inesul.edu.br/maranhao/mat3.htm $>$.Acesso em:10 de novembro de 2020.

LOPES, P. A. Entendendo a importância da estatística sem ser gênio, matemático ou bruxo. 2005. Disponível em: <http://www.administradores.com.br/informe-se/artigos/ entendendo-a-importancia-da-estatistica-sem-ser-genio-matematico-ou-bruxo/11591/>. Acesso em: 15 de novembro de 2020.

MAKRYMICHALOS, M. et al. Statisticalthinkingand its role for industrial engineersand managers in the 21st century. ManagerialAuditingJournal, v. 20, n. 4, p. 351-

363, 2005.Disponível em: <http://dx.doi.org/10.1108/02686900510592043>. Acesso em: 31 de maio de 2021.

MATSUSHITA, R. Y. O que é estatística? Disponível em: $<$ http://vsites.unb.br/ie/est/ complementar/estatistica.htm>. Acesso em: 14 de novembro de 2020.

MORETTIN, P. A. Introdução à estatística para ciências exatas. São Paulo: Atual, 1981.

NORA, J.A.F. Análise da transição para um sistema de gestão da qualidade ISSO

9001:2000 na Indústria de Material Bélico do Brasil. Dissertação (Mestrado emEngenharia de Produção) - Universidade Federal de Itajubá, Itajubá, 2003.

PIMENTEL, A. Estatística é base para previsões meteorológicas. Diário do Nordeste, 25 jan. 2009. Disponível em: $<$ http://diariodonordeste.globo.com/materia.asp?codigo=609209>. Acesso em: 17 de novembro de 2020.

RAO, C. R. Statisticsandtruth:putting chance towork. 2nd. ed. Singapore: World Scientific, 1997. 
SALSBURG, D. Uma senhora toma chá...: como a estatística revolucionou a ciência no século XX. Rio de Janeiro: Zahar, 2009.

SANTOS, A. B.; MARTINS, M. F. Modelo de referência para estruturar o Seis Sigma nas organizações. Gestão \& Produção, v. 15, n. 1, p. 43-56, 2008. Disponível em:

$<$ http://dx.doi.org/10.1590/S0104-530X2008000100006>. Acesso em: 31 de maio de 2021.

STIGLER, S. M. The historyofstatistics: themeasurementofuncertaintybefore 1900.

Cambridge: Belknap Press of Harvard University Press, 1986.

YANG, J. Managingknowledge for qualityassurance: anempiricalstudy.

InternationalJournalofQualityandReliability Management, v. 25, n. 2, p. 109-124, 2008.

YIN, Robert K. Estudo de caso: planejamento e métodos. 2. ed. Porto Alegre:

Bookman,2001. 